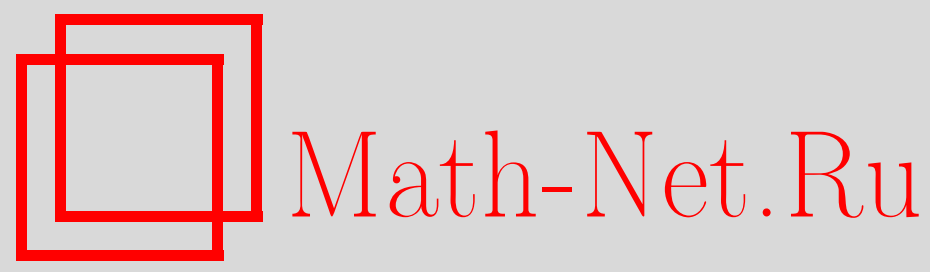

С. Г. Крыжевич, Об асимптотических свойствах решений систем с малой нелинейностью в окрестности особой точки, Матем. заметки, 2004, том 75, выпуск 5, 683-692

DOI: https://doi.org/10.4213/mzm64

Использование Общероссийского математического портала Math-Net.Ru подразумевает, что вы прочитали и согласны с пользовательским соглашением http://www.mathnet.ru/rus/agreement

Параметры загрузки:

IP: 18.234 .156 .22

26 апреля 2023 г., $16: 56: 28$ 


\section{ОБ АСИМПТОТИЧЕСКИХ СВОЙСТВАХ РЕШЕНИЙ СИСТЕМ С МАЛОЙ НЕЛИНЕЙНОСТЬЮ В ОКРЕСТНОСТИ ОСОБОЙ ТОЧКИ \\ С. Г. Крыжевич}

Рассматриваются классы так называемых слабо гиперболичных линейных систем, включающие в себя как правильные, так и гиперболичные (экспоненциально-дихотомичные) системы. Получены результаты об условной устойчивости решений, обобщающие ряд классических теорем. Показывается существование устойчивых поверхностей, точкам которых соответствуют решения исходной системы с отрицательными показателями Ляпунова. Исследуется поведение решений, начинающихся вне устойчивых поверхностей. Используются методы, схожие с теми, что применяются в теории гиперболических систем.

Библиография: 7 названий.

Изучается задача о поведении решений нелинейной системы в окрестности точки покоя. Рассмотрим систему

$$
\dot{x}=A(t) x
$$

размерности $n$ с кусочно-непрерывными при $t \geqslant t_{0}$ матрицами коэффициентов и систему

$$
\dot{x}=A(t) x+f(t, x),
$$

получаемую из (0.1) добавлением нелинейных возмущений $f(t, x)$, удовлетворяющих условию $f(t, 0) \equiv 0$.

Пусть пространство решений системы (0.1), экспоненциально стремяшихся к нулю при $t \rightarrow+\infty$, имеет размерность $k$. Возникает вопрос: при каких условиях на матрицу $A$ и нелинейность $f$ для системы $(0.2)$ найдется $k$-мерное многообразие начальных данных при $t=t_{0}$, которым соответствуют решения, экспоненциально стремящиеся $\mathrm{k}$ нулю при $t \rightarrow \infty$. В случае, когда система (0.1) правильна, а нелинейность $f$ аналитична и ее ряд Тейлора начинается с членов второго порядка малости, существование устойчивого многообразия было установлено А. М. Ляпуновым [1]. Для систем с гиперболическим линейным приближением и возмущением $f$, удовлетворяющим в окрестности нуля условию Липшица с малой константой, аналогичньй результат был установлен О. Перроном [2] (см. также [3, гл. 1]). Д. М. Гробман [4] получил аналогичный результат для систем с правильной линейной частью и экспоненциально стремящейся к нулю

Работа выполнена при частичной поддержке Российского фонда фундаментальных исследований, грант № 99-01-00683, программы "Ведущие научные школы", грант № 00-15-96021, а также Министерства Образования России и Правительства Санкт-Петербурга, грант № PD03-1.1-142. 
нелинейностью. В статьях [5] и [6] автору удалось, введя новые классы слабо гиперболических линейных систем, включаюшие в себя гиперболические и правильные системы, доказать теоремы об условной устойчивости по первому приближению, обобщающие результаты Ляпунова, Перрона и Гробмана. Формулировки этих теорем приведены в разделах 1 и 2 настоящей статьи.

Основные результаты содержатся в разделе 3. В статье Перрона показано, что все решения системы с гиперболической линейной частью, начинающиеся вне устойчивого многообразия, покидают с течением времени достаточно малую окрестность начала координат. Оказывается, при различных предположениях на нелинейное возмущение $f$, аналогичные результаты можно получить и для систем со слабо гиперболичной линейной частью. Это дает мощньй инструмент для вычисления характеристических показателей решений нелинейных систем.

1. Определение и свойства слабо гиперболичных систем. Обозначим символом $\|\cdot\|$ некоторую векторную норму в $\mathbb{R}^{n}$ и соответствующую ей матричную норму. Условимся считать, что $\|x\|=\max \left(\left|x_{i}\right|\right)$, где $x=\operatorname{col}\left(x_{1}, \ldots, x_{n}\right)$. Обозначим также через $\chi(\cdot)$ характеристический показатель Ляпунова определенный для функций, вектор-функций и матриц по следующей формуле [7]:

$$
\chi(f)=\limsup _{t \rightarrow+\infty} \frac{1}{t} \ln \|f(t)\| .
$$

ОПРЕДЕЛЕНИЕ 1.1. Назовем систему (0.1) слабо гиперболичной с константами $\lambda>0$ и $\varepsilon \geqslant 0$, если существует такое число $K>0$, что для любой измеримой вектор-функции $g:\left[t_{0}, \infty\right) \rightarrow \mathbb{R}^{n}$, удовлетворяющей при почти всех $t \geqslant t_{0}$ оценке

$$
\|g(t)\| \leqslant \exp (-\lambda(1+\varepsilon) t)
$$

у линейной неоднородной системы

$$
\dot{x}=A(t) x+g(t)
$$

существует решение $\varphi(t)$ такое, что

$$
\|\varphi(t)\| \leqslant K \exp (-\lambda t) \quad \text { при } t \geqslant t_{0} .
$$

Обозначим введенный вьше класс систем через $\mathrm{WH}(\lambda, \varepsilon)$.

ТЕОРема 1.1. Пусть система (0.1) гиперболична (әкспоненциально-дихотомична). Тогда существует такое число $\lambda_{0}>0$, что эта система принадлежит классу $\mathrm{WH}(\lambda, 0)$ при любом выборе $\lambda \in\left(0, \lambda_{0}\right)$.

ТЕОремА 1.2. Если система (0.1) имеет коэффициент неправильности Гробмана, меньиий $\lambda \varepsilon$ для некоторых положительных $\lambda$ и $\varepsilon$, то данная система принадлежит классу $\mathrm{WH}(\lambda, \varepsilon)$.

Для любого числа $\mu>0$ обозначим через $\exp (-\mu t) \mathbb{L}^{\infty}$ пространство вектор-функций, представимых в виде произведения ограниченной измеримой вектор-функции на $\exp (-\mu t)$. Введем норму в пространствах $\exp (-\mu t) \mathbb{L}^{\infty}$ по формуле

$$
\|g\|=\underset{t \geqslant t_{0}}{\operatorname{ess} \sup }(\exp (\mu t)\|g(t)\|)
$$


ТЕОРема 1.3. Пусть система (0.1) слабо гиперболична с некоторыми константами $\lambda$ и $\varepsilon$. Тогда существуют число $C>0$ и непрерьвньй линейный оператор

$$
\mathscr{L}: \exp (-\lambda(1+\varepsilon) t) \mathbb{L}^{\infty} \rightarrow \exp (-\lambda t) \mathbb{L}^{\infty}
$$

такие, что если выполняется неравенство

$$
\|g(t)\| \leqslant \exp (-\lambda(1+\varepsilon) t)
$$

то функиия $\mathscr{L} g(t)$ является решением системы (1.1) $и$

$$
\|\mathscr{L} g(t)\| \leqslant C \exp (-\lambda t)
$$

2. Условная устойчивость нулевых решений систем со слабо гиперболичной линейной частью. Выберем некоторьй мультииндекс $l=\left(l_{1}, \ldots, l_{n}\right)$ и вектор $x=\operatorname{col}\left(x_{1}, \ldots, x_{n}\right)$. Введем следуюшие обозначения:

$$
|l|=\sum_{i=1}^{n} l_{i}, \quad l !=l_{1} ! \cdots l_{n} !, \quad x^{l}=x_{1}^{l_{1}} \cdots x_{n}^{l_{n}}, \quad \frac{\partial^{|l|} f}{\partial x^{l}}=\frac{\partial^{|l|} f}{\partial x_{1}^{l_{1}} \cdots \partial x_{n}^{l_{n}}} .
$$

ОПРЕДЕЛЕниЕ 2.1. Пусть $U$ - некоторая окрестность начала координат в $\mathbb{R}^{n}$,

$$
f:\left[t_{0}, \infty\right) \times U \rightarrow \mathbb{R}^{n}
$$

- отображение, непрерьвное по $t$ и $C^{1}$-гладкое по $x, \alpha>1$ - вещественное число и $N-$ наибольшее натуральное число, строго меньшее $\alpha$. Будем говорить, что отображение $f$ имеет порядок малости $\alpha$ по $x$, если в окрестности $U$ выполнены следуюшие условия:

1) $f(t, 0)=0$ для любых $t \geqslant t_{0}$;

2) $f \in C_{x}^{N}$ и для любого мультииндекса $l$ такого, что $|l| \leqslant N$, выполнено

$$
\frac{\partial^{|l|} f}{\partial x^{l}}(t, 0)=0
$$

3) сушествует число $L>0$ такое, что для любого мультииндекса $l$, для которого $|l|=N$, и произвольного $t \geqslant t_{0}$ вьполнено условие Гёльдера: для любых $x_{1}, x_{2} \in U$

$$
\left\|\frac{\partial^{|l|} f}{\partial x^{l}}\left(t, x_{1}\right)-\frac{\partial^{|l|} f}{\partial x^{l}}\left(t, x_{2}\right)\right\| \leqslant L\left\|x_{1}-x_{2}\right\|^{\alpha-N} .
$$


ТЕОремА 2.1. Пусть числа $\lambda, \sigma$ и в положительны, а система (0.1) принадлежит классу $\mathrm{WH}(\lambda, \varepsilon)$. Предположим, что для системы $(0.2)$, определенной на множестве

$$
\Omega=\left\{t, x: t \geqslant t_{0},\|x\|<\sigma\right\},
$$

выполнены следующие условия:

1) отображение $f$ имеет порядок малости не менее $1+\varepsilon$ по $x$ в некоторой окрестности $U$ начала координат в $\mathbb{R}^{n}$;

2) если $\lambda_{1}, \ldots, \lambda_{n}$ - характеристические показатели системы (0.1), то для некоторого $0 \leqslant k \leqslant n$ справедливы неравенства

$$
\lambda_{1} \leqslant \cdots \leqslant \lambda_{k}<-\lambda<\lambda_{k+1} \leqslant \cdots \leqslant \lambda_{n}
$$

Если системы (0.1) и (0.2) удовлетворяют перечисленным выше условиям, а $M$ - наибольшее натуральное число, строго меньшее $1+\varepsilon$, то существует $C^{M}$-гладкое и взаимно однозначное отображение $g$ окрестности нуля из $\mathbb{R}^{k}$ в $\mathbb{R}^{n}$ такое, что

1) $g(0)=0$;

2) если $\Phi(t)=\left(X_{1}(t), \ldots, X_{n}(t)\right)$ - нормальная фундаментальная система решений (0.1) такая, что характеристические показатели вектор-функиий

$$
X_{1}(t), \ldots, X_{n}(t)
$$

равны $\lambda_{1}, \ldots, \lambda_{n}$ соответственно, а $J$-матрица Якоби отображсения $g$ в точке 0, mо $J=\left(X_{1}\left(t_{0}\right), \ldots, X_{k}\left(t_{0}\right)\right)$;

3) для любого $x_{0}$, для которого $\left\|x_{0}\right\|<\sigma$, и для которого существует вектор $y_{0} \in \mathbb{R}^{k}$ такой, что $x_{0}=g\left(y_{0}\right)$, решение системы $(0.2)$ с начальными данными $x\left(t_{0}\right)=x_{0}$ имеет при $t \rightarrow+\infty$ характеристический показатель, не превосходящий $-\lambda$.

Полностью доказательство этого утверждения, равно как и теоремы 2.2, изложено в работе [6]. Отметим, однако, некоторые моменты доказательства, необходимые для дальнейших рассуждений.

Построим приближенные решения системы (0.2) по следующему алгоритму. Возьмем некоторьй достаточно мальй по норме вектор $y=\operatorname{col}\left(y_{1}, \ldots, y_{k}\right) \in \mathbb{R}^{k}$ и введем в рассмотрение последовательность вектор-функций

$$
\left\{X^{(m)}(t, y)\right\}=X^{(1)}(t, y), X^{(2)}(t, y), \ldots, X^{(m)}(t, y), \ldots
$$

Первьй ее член представи́м в виде линейной комбинации столбцов матрицы $\Phi(t)$ с характеристическими показателями, меньшими числа $-\lambda$ :

$$
X^{(1)}(t, y)=\sum_{r=1}^{k} y_{r} X_{r}(t)
$$

Вектор-функции $X^{(m)}(t, y)$ последовательно определяются по формулам

$$
X^{(m+1)}(t, y)=X^{(1)}(t, y)+\mathscr{L}\left[f\left(\cdot, X^{(m)}(\cdot, y)\right)\right](t) .
$$

В предположении $X^{(0)}(t, y) \equiv 0$ была доказана следующая лемма. 
ЛЕМма 2.1. Пусть

$$
D=\sup _{t \geqslant t_{0}} \max _{i \leqslant k}\left\|X_{i}(t)\right\| \exp (\lambda t)
$$

Для любого $q \in(0,1)$ существует $\delta>0$ такое, что если $\|y\| \leqslant \delta$, то последовательные приближения $X^{(2)}(t, y), X^{(3)}(t, y), \ldots, X^{(m)}(t, y), \ldots$, получаемые по формулам (2.3), определены и имеют характеристические показатели, не большие $-\lambda$. Для любых $m \geqslant 1, t \geqslant t_{0}$ и произвольных векторов у таких, что $\|y\| \leqslant \delta$, верна оченка

$$
\left\|X^{(m)}(t, y)-X^{(m-1)}(t, y)\right\| \leqslant D q^{m-1} \exp (-\lambda t)\|y\|, \quad m \geqslant 2 .
$$

Итак, последовательность $X^{(m)}(t, y)$ сходится к непрерывной функции $X^{*}(t, y)$ равномерно по всем $t \in\left[t_{0}, \infty\right)$, причем при достаточно малых по норме $y$ функции $X^{*}(\cdot, y)$ являются решениями системы (0.2) с характеристическими показателями, не превосходящими $-\lambda$. Отображение $g$ из условия теоремы можно определить по формуле $g(t)=$ $X^{*}\left(t_{0}, y\right)$.

Рассмотрим систему (0.2) с таким возмущением $f(t, x)$, что $f(t, 0) \equiv 0$, и вместо рассмотренного вьше условия малости на множестве $\Omega$ из условия теоремы 2.1 вьполнено условие Липшища

$$
\left\|f\left(t, x_{1}\right)-f\left(t, x_{2}\right)\right\| \leqslant L(t)\left\|x_{1}-x_{2}\right\|
$$

для любых $t \geqslant t_{0}, x_{1}, x_{2} \in U$.

ТЕОрема 2.2. Пусть $\lambda>0, \varepsilon \geqslant 0$, система (0.1) принадлежит классу $\mathrm{WH}(\lambda, \varepsilon)$, и число $k$ таково, что ее характеристические показатели удовлетворяют (2.2). Пусть, кроме того, для возмущения $f$ выполнено условие (2.6), причем

$$
L(t)<l \exp (-\lambda \varepsilon t)
$$

Тогда найдется такое $l_{0}>0$, что для всех $0<l \leqslant l_{0}$ существует непрерывное отображсение $h$ некоторой окрестности нуля из $\mathbb{R}^{k}$ в $\mathbb{R}^{n}$ такое, что

1) $h(0)=0$

2) $\left\|h\left(y_{1}\right)-h\left(y_{2}\right)\right\| \leqslant D l\left\|y_{1}-y_{2}\right\|$, причем константа $D$ зависит только от свойств системы $(0.1)$;

3) если $J=\left(X_{1}\left(t_{0}\right), \ldots, X_{k}\left(t_{0}\right)\right)$, то для любого $x_{0}$ такого, что $\left\|x_{0}\right\|<\sigma$, для которого существует такое $y_{0} \in \mathbb{R}^{k}$, ито $x_{0}=g\left(y_{0}\right)=J y_{0}+h\left(y_{0}\right)$, pешение системь (0.2) с начальными данными $x\left(t_{0}\right)=x_{0}$ имеет при $t \rightarrow+\infty$ характеристический показатель, не превосходящий $-\lambda$.

В основе доказательства лежит метод последовательных приближений (2.3). Оказывается, что при малых $l$ все последовательные приближения определены и при любых $m$ удовлетворяют оценкам

$$
\left\|X^{(m)}(t, y)\right\| \leqslant D\|y\| \exp (-\lambda t)
$$

где

$$
D=2 C \sup _{\substack{t \geqslant t_{0} \\ 0 \neq y \in \mathbb{R}^{k}}} \frac{\left\|X^{(1)}(t, y)\right\|}{\|y\|} \exp (\lambda t) .
$$


3. Поведение решений возмущенной системы, начинающихся вне устойчивого многообразия. Пусть система (0.1) принадлежит классу $\mathrm{WH}(\lambda, \varepsilon)$. Обозначим через $U^{+}(s)$ пространство значений при $t=s$ решений системы $(0.1)$, имеющих характеристический показатель, меньший $-\lambda$. Пусть, кроме того, для системы $(0.2)$ выполнены условия теоремы 2.1 или теоремы 2.2 , причем система $(0.2)$ во всей своей области определения удовлетворяет условию существования и единственности решений.

ОПРЕДЕЛЕниЕ 3.1. Определим для системы (0.2), пользуясь обозначениями теоремы 2.1, многообразие, устойчивое в положительном направлении, по формуле

$$
W_{\text {loc }}^{s}\left(t_{0}, \lambda, \varepsilon\right)=\left\{x_{0} \in \mathbb{R}^{n}:\left\|x_{0}\right\|<\sigma, x_{0}=g\left(y_{0}\right) \text { для некоторого } y_{0}\right\} .
$$

Обозначим для любых $t, \tau \geqslant t_{0}, x_{0} \in U$ символом $x\left(t, \tau, x_{0}\right)$ решение системы $(0.2)$ с начальньми данными $x(\tau)=x_{0}$. Определим глобальное устойчивое многообразие системы (0.2) по формуле

$$
W^{s}\left(t_{0}, \lambda, \varepsilon\right)=\bigcup_{t \geqslant t_{0}}\left\{x_{0} \in \mathbb{R}^{n}: x\left(t, t_{0}, x_{0}\right) \in W_{\mathrm{loc}}^{s}(t, \lambda, \varepsilon)\right\} .
$$

Рассмотрим для произвольного $t \geqslant t_{0}$ отображение $g_{t}: V_{t} \rightarrow \mathbb{R}^{n}$, определенное по формуле

$$
g_{t}(y)=X^{*}(t, y)
$$

существующее в силу теоремы 2.1 или теоремы 2.2 .

Заметим, что из построения отображений $g_{t}$ следует, что

$$
g_{t}(y)=x\left(t, \tau, g_{t_{0}}(y)\right)
$$

для любых $t \geqslant \tau \geqslant t_{0}, y \in \mathbb{R}^{k}$, для которых определена правая часть этого равенства. Таким образом, можно доопределить отображение $g=g_{t_{0}}$ следующим образом. Выбираем для фиксированного $y \in \mathbb{R}^{k}$ число $T$ столь большим, что отображения $g_{t}(y)$ были определены для любых $t \geqslant T$ (если такое число $T$ для этого $y$ существует). Положим

$$
g(y)=x\left(t_{0}, T, g_{T}(y)\right)
$$

если выражение в правой части этого равенства определено (данное решение может покинуть с убыванием времени множество $U$, где определена система (0.2)). Аналогичным образом можно доопределить отображения $g_{t}$ для любого $t \geqslant t_{0}$.

Исследуем поведение решений, не проходяших при $t=t_{0}$ через $W^{s}\left(t_{0}, \lambda, \varepsilon\right)$. В последующих рассуждениях будем использовать те же идеи, что применяются при исследовании гиперболических систем.

ЛЕмма 3.1. Пусть для системы (0.2) выполнены условия теоремы 2.2, где число $l$ достаточно мало. Тогда для любого числа $\tau \geqslant t_{0}$ и любого вектора $c \in U^{+}(\tau)$ система

$$
x(t)=\Phi(t, \tau) c+\mathscr{L}[f(\cdot, x(\cdot))](t)
$$

имеет не более одного решения $\varphi(t)$, для которого функиия $\exp (\lambda t)\|\varphi(t)\|$ ограничена при $t \geqslant \tau$. 
ДокАЗАТЕЛЬСТво. Предположим, что $\varphi_{1}(t)$ и $\varphi_{2}(t)$ - два решения $(3.1)$, удовлетворяющих условиям леммы. Введем обозначение

$$
\alpha=\sup _{t \geqslant \tau} \exp (\lambda t)\left\|\varphi_{1}(t)-\varphi_{2}(t)\right\| .
$$

По условию $0 \leqslant \alpha<+\infty$. Поскольку $\varphi_{1}(t)$ и $\varphi_{2}(t)$ - решения системы $(3.1)$, вьполнено $\varphi_{i}(t)=\Phi(t, \tau) c+\mathscr{L}\left[f\left(\cdot, \varphi_{i}(\cdot)\right](t)\right.$ при $i \in\{1,2\}$, откуда следует, что

$$
\varphi_{1}(t)-\varphi_{2}(t)=\mathscr{L}\left[f\left(\cdot, \varphi_{1}(\cdot)\right)-f\left(\cdot, \varphi_{2}(\cdot)\right)\right](t) .
$$

Из формул (2.6) и (2.7) следует, что для любого $t \geqslant \tau$

$$
\left\|f\left(t, \varphi_{1}(t)\right)-f\left(t, \varphi_{2}(t)\right)\right\| \leqslant l \exp (-\lambda \varepsilon t)\left\|\varphi_{1}(t)-\varphi_{2}(t)\right\| \leqslant l \alpha \exp (-\lambda(1+\varepsilon) t),
$$

откуда вытекает, что $\left\|\varphi_{1}(t)-\varphi_{2}(t)\right\| \leqslant C l \alpha \exp (-\lambda t)$, где $C$ - норма оператора $\mathscr{L}$. Но если число $l$ было выбрано таким, что $C l \leqslant 1 / 2$, то $\left\|\varphi_{1}(t)-\varphi_{2}(t)\right\| \exp (\lambda t) \leqslant \alpha / 2$. Переходя в последнем неравенстве к супремуму по $t \geqslant \tau$, получаем, что $\alpha \leqslant \alpha / 2$ и, следовательно, $\alpha=0$, так как $\alpha \geqslant 0$. Значит, $\varphi_{1}(t)=\varphi_{2}(t)$, что и требовалось доказать.

ЛЕмма 3.2. Для любой системы (0.2), удовлетворяющей условиям теоремы 2.1, существует $\beta>0$ такое, что для любых $\tau \geqslant t_{0}$ и с $\in U^{+}(\tau)$ система (3.1) имеет не более одного решения $\varphi(t)$, для которого

$$
\|\varphi(t)\| \leqslant \beta \exp (-\lambda t) .
$$

ДокАЗАТЕЛЬСтво. Выберем $\varphi_{1}(t), \varphi_{2}(t)$ и $\alpha$ по аналогии с доказательством предыдущей леммы. Отметим, что, так как $\left\|\varphi_{i}(t)\right\| \leqslant \beta \exp (-\lambda t)$, справедливо неравенство

$$
\alpha \leqslant 2 \beta \text {. }
$$

Из формулы Тейлора для вектор-функции $f$ получаем

$$
\begin{aligned}
\left\|f\left(t, \varphi_{1}(t)\right)-f\left(t, \varphi_{2}(t)\right)\right\| \leqslant & \sum_{r=1}^{M-1} \sum_{|l|=r} \frac{n^{M-r}}{l !} L\left\|\varphi_{2}(t)\right\|^{1+\varepsilon-r}\left\|\varphi_{1}(t)-\varphi_{2}(t)\right\|^{r} \\
& +\sum_{|l|=M} \frac{1}{l !} L \max \left\{\left\|\varphi_{1}(t)\right\|,\left\|\varphi_{2}(t)\right\|\right\}^{1+\varepsilon-M}\left\|\varphi_{1}(t)-\varphi_{2}(t)\right\|^{M} \\
\leqslant & \sum_{r=1}^{M} \sum_{|l|=r} L \frac{n^{M-r}}{l !} \beta^{1+\varepsilon-r} \alpha^{r} \exp (-\lambda(1+\varepsilon) t) \\
\leqslant & \beta^{\varepsilon} \alpha L \sum_{r=1}^{M \text { कормуле }(3.3)} n^{M-r} 2^{r-1} \sum_{|l|=M} \frac{1}{l !} \exp (-\lambda(1+\varepsilon) t),
\end{aligned}
$$

откуда

$$
\left\|\mathscr{L}\left[f\left(\cdot, \varphi_{1}(\cdot)\right)-f\left(\cdot, \varphi_{2}(\cdot)\right)\right](t)\right\| \leqslant \widetilde{C} \beta^{\varepsilon} \alpha \exp (-\lambda t),
$$

где

$$
\widetilde{C}=C L \sum_{r=1}^{M} n^{M-r} 2^{r-1} \sum_{|l|=r} \frac{1}{l !} .
$$

Тогда, выбирая достаточно малое $\beta$, получаем $\left\|\varphi_{1}(t)-\varphi_{2}(t)\right\| \leqslant \alpha \exp (-\lambda t) / 2$, откуда, как и при доказательстве леммы 3.1 , следует, что $\varphi_{1}(t) \equiv \varphi_{2}(t)$. Лемма 3.2 доказана. 
ТЕОРемА 3.1. Пусть для системы (0.2) выполнены условия теоремы 2.1 или теоремы 2.2. Тогда существуют $\delta, \beta>0$ такие, что любое решение $\psi(t)$ системы (0.2), начинающееся при $t=t_{0}$ в $U_{\delta}-\delta$-окрестности нуля в $\mathbb{R}^{n}, и$ вне поверхности $W^{s}\left(t_{0}, \lambda, \varepsilon\right)$, не удовлетворяет при некотором $t \geqslant t_{0}$ оценке

$$
\|\psi(t)\|<\beta \exp (-\lambda t)
$$

или не продолэимм на луч $\left[t_{0},+\infty\right)$.

ДокАЗАТЕльство. Пусть $\psi(t)$ - некоторое решение системы $(0.2)$, удовлетворяющее оценке (3.7) при всех $t \geqslant t_{0}$. Тогда справедливо тождество $\dot{\psi}(t)=A(t) \psi(t)+$ $f(t, \psi(t))$, откуда следует, что $\psi(t)$ есть решение линейной неоднородной системы

$$
\dot{x}=A(t) x+f(t, \psi(t))
$$

Поскольку справедливо (3.7), из (3.8) следует, что найдется такой вектор $c_{0} \in U^{+}\left(t_{0}\right)$, что $\psi(t)=\Phi\left(t, t_{0}\right) c_{0}+\mathscr{L}[f(\cdot, \psi(\cdot))](t)$. Таким образом, $\psi(t)$ является решением системы (3.1) для $\tau=t_{0}, c=\mathrm{C}_{0}$.

Положим $y_{0}=J^{-1} c_{0}$. В данном случае $J: \mathbb{R}^{k} \rightarrow U^{+}\left(t_{0}\right)$ рассматривается как обратимьй линейный оператор, представляющий собой умножение первых $k$ столбцов матрицы $\Phi\left(t_{0}\right)$ на $k$-мерньй вектор.

Заметим, что число, обозначенное буквой $D$ и определенное при доказательстве теоремы 2.1 по формуле (2.4) (равно как и то, что определялось при доказательстве теоремы 2.2 по формуле (2.9)), экспоненциально убьвает с ростом $t_{0}$. Кроме того, вектор-функция $\Phi\left(t, t_{0}\right) c_{0}$ стремится к нулю с ростом $t$. Повторяя рассуждения теоремы 2.1 и теоремы 2.2 , можно показать, что существует число $\tau \geqslant t_{0}$ такое, что при $t \geqslant \tau$ и $y$, близких к $y_{0}$, сходимость метода последовательных приближений будет обеспечена (она может быть обусловлена не только малостью вектора $y$, но и малостью значения $D$ вкупе с малостью начального приближения).

Положим $c=J_{\tau}\left(y_{0}\right)$. Тогда система (3.1) имеет решение $\varphi(t)$, определенное при $t \geqslant \tau$ и удовлетворяющее условию (3.2), причем $\varphi(\tau)=g_{\tau}\left(y_{0}\right)$. Но $\psi(t)$ при $t \geqslant \tau$ также является решением (3.1). Из леммы 3.2 (если нелинейная система удовлетворяет условиям теоремы 2.1) или леммы 3.1 (если мы находимся в условиях теоремы 2.2) следует, что $\psi(t) \equiv \varphi(t)$ при $t \geqslant \tau$. Тогда по определению устойчивого многообразия $\psi\left(t_{0}\right) \in W^{s}\left(t_{0}, \lambda, \varepsilon\right)$. Теорема доказана.

Из теоремы 3.1 вытекает следующий результат.

ТЕОрема 3.2. Пусть для системы (0.2) выполнены условия теоремы 2.1 и, кроме того, справедливо одно из следующих утвержсдений:

1) система линейного приближения (0.1) принадлежит классу $W H(\bar{\lambda}, \varepsilon)$ для некоторого $\bar{\lambda} \in\left(\lambda,-\lambda_{k}\right)$;

2) отображение $f$ имеет порядок малости $1+\bar{\varepsilon}$ для некоторого $\bar{\varepsilon}>\varepsilon$.

Тогда существует $\delta>0$ такое, что если решение $\psi(t)$ системы (0.2) начинается при $t=t_{0}$ в $U_{\delta}$ вне поверхности $W^{s}\left(t_{0}, \lambda, \varepsilon\right)$ и продолэимо на луч $\left[t_{0},+\infty\right)$, то функиия $\exp (\lambda t)\|\psi(t)\|$ не ограничена при $t \geqslant t_{0}$. 
ДокАЗАТЕльство. Предположим сначала, что вьполнено утверждение п. 1). Как следует из доказательства леммы 3.2 (формулы (3.5) и (3.6)), число $\beta$ в условии леммы 3.1 может быть выбрано независимо от значения $t_{0}$. Таким образом, можно выбрать сколь угодно большое $t$, для которого оценка (3.7) несправедлива. Заменяя $\lambda$ на $\bar{\lambda}$ и применяя теорему 3.1 с указанным выше замечанием, получаем стремящуюся к бесконечности последовательность $\left\{t_{n}\right\}$ такую, что $\left\|\psi\left(t_{n}\right)\right\| \geqslant \beta \exp \left(-\bar{\lambda} t_{n}\right)$. А тогда последовательность $\exp \left(\lambda t_{n}\right)\left\|\psi\left(t_{n}\right)\right\|$ неограничена, откуда вытекает справедливость доказьваемого утверждения.

Пусть теперь справедливы условия п. 2). Докажем следующую лемму.

Лемма 3.3. В условиях $n$. 2) доказываемой теоремы существует $\delta>0$ такое, что для любого вектора $c \in U^{+}\left(t_{0}\right)$, норма которого меньше $\delta$, система (3.1) имеет ровно одно решение $\varphi(t)$, для которого функиия $\exp (\lambda t)\|\varphi(t)\|$ ограничена npu $t \geqslant t_{0}$.

ДокАЗАТЕЛЬСТвО. Пусть вопреки предположению для некоторого вектора $c$, удовлетворяющего приведенным условиям, существуют два решения указанного вида - $\varphi_{1}(t)$ и $\varphi_{2}(t)$. Пусть число $\beta$ таково, что справедливо утверждение теоремы 3.1. Выберем $t_{1} \geqslant t_{0}$ столь большим, что $\widetilde{C} \beta^{\bar{\varepsilon}} \exp \left(-\lambda(\bar{\varepsilon}-\varepsilon) t_{1}\right) \leqslant 1 / 2$, где число $\widetilde{C}$ определяется по формуле (3.6). Положим

$$
\alpha=\sup _{t \geqslant t_{1}} \exp (\lambda t)\left\|\varphi_{1}(t)-\varphi_{2}(t)\right\|
$$

При $t \geqslant t_{0}$ справедливо неравенство, получающееся заменой $\varepsilon$ на $\bar{\varepsilon}$ в правой части (3.4), а именно,

$$
\left\|f\left(t, \varphi_{1}(t)\right)-f\left(t, \varphi_{2}(t)\right)\right\| \leqslant \beta^{\bar{\varepsilon}} \alpha L \sum_{r=1}^{M} n^{M-r} 2^{r-1} \sum_{|l|=r} \frac{1}{l !} \exp (-\lambda(1+\bar{\varepsilon}) t) .
$$

Так как $\exp (-\lambda(1+\bar{\varepsilon}) t) \leqslant \exp \left(-\lambda(\bar{\varepsilon}-\varepsilon) t_{1}\right) \exp (-\lambda(1+\varepsilon) t)$ при $t \geqslant t_{1}$, а $\beta^{\bar{\varepsilon}}<\beta^{\varepsilon}$ при $\beta<1$, из (3.9) вытекает справедливость неравенства

$$
\left\|\mathscr{L}\left[f\left(\cdot, \varphi_{1}(\cdot)\right)-f\left(\cdot, \varphi_{2}(\cdot)\right)\right](t)\right\| \leqslant \widetilde{C} \beta^{\varepsilon} \exp \left(-\lambda(\bar{\varepsilon}-\varepsilon) t_{1}\right) \alpha \exp (-\lambda t) \leqslant \frac{\alpha \exp (-\lambda t)}{2} .
$$

Лемма доказана.

Далее, по аналогии с доказательством теоремы 3.1 получаем справедливость утверждения доказьваемой теоремы.

Отметим важное следствие полученных результатов.

СлЕДСТВИЕ 3.1. Пусть матрица $A(t)$ коэффициентов системы (0.1) ограничена, а гробмановский коэффициент неправильности этой системы равен $\sigma$. Пусть нелинейность $f(t, x)$ имеет порядок малости $1+\varepsilon$ по $x$. Обозначим через $\lambda_{1} \leqslant \cdots$ $\leqslant \lambda_{k}$ множсество характеристических показателей системы (0.1), меньиих чис-

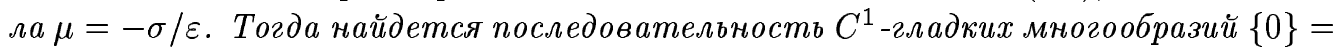
$W_{0} \subset W_{1} \subset \cdots \subset W_{k}=W^{s}$, удовлетворяющих следующим условиям:

1) $\operatorname{dim} W_{i}=i$ для любого $i$ от 1 до $k$;

2) если $\Phi(t)=\left(\varphi_{1}(t), \ldots, \varphi_{n}(t)\right)$ - фундаментальная матрица системы $(0.2)$ такая, ито $\chi\left(\varphi_{i}(t)\right)=\lambda_{i}, i=1, \ldots, n, \operatorname{mo} T W_{i}(0)=\operatorname{Lin}\left(\varphi_{1}(0), \ldots, \varphi_{i}(0)\right)$;

3) если $x_{0} \in W_{i} \backslash W_{i-1}$, то $\chi\left(x\left(t, 0, x_{0}\right)\right)=\lambda_{i}$. 
Приведенньй результат может быть использован для точного вычисления характеристических показателей решений нелинейных систем.

СлЕДСТВИЕ 3.2. Множество отрицательных характеристических показателей нелинейной системы с правильной линейной частью и возмущением порядка малости больше единииь совпадает с соответствующим мнохеством для линейной системы первого приближения.

\section{СПИСОК ЦИТИРОВАННОЙ ЛИТЕРАТУРЫ}

[1] Ляпунов А. М. Общая задача об устойчивости движения. Т. 2. М., 1956.

[2] Perron O. Über Stabilitat und asymptotisches Verhalten der Integrale von Differentialgleichungssystemen // Math. Z. 1929. V. 29. P. 129-160.

[3] Плисс В. А. Интегральные множества периодических систем дифференциальных уравнений. М.: Наука, 1977.

[4] Гробман Д. М. Характеристические показатели систем, близких к линейным // Матем. сб. 1952. Т. 30. №1. С. 121-166.

[5] Крыжевич С. Г. Условная устойчивость систем обыкновенных дифференциальных уравнений и полугиперболические системы // Дифференц. уравнения. 2000. Т. 36. №1. С. 39-47.

[6] Крыжевич С. Г. Интегральная разделенность и условная устойчивость решений обькновенных дифференциальных систем по первому приближению // Вестн. молодых ученых. Сер. прикл. матем. и мех. 2000. № 3. С. 34-49.

[7] Адрианова Л.Я. Введение в теорию линейных систем дифференциальных уравнений. С.-Пб.: Изд-во С.-Петербургского ун-та, 1992.

Санкт-Петербургский государственный университет

Поступило

E-mail: sgk@fromru.com, kryzhevitz@rambler.ru

16.11.2001

Исправленный вариант

18.06 .2003 